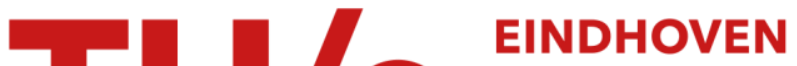 UNIVERSITY OF TECHNOLOGY
}

\section{Towards a Scaleable 5G Fronthaul: Analog Radio-over-Fiber and Space Division Multiplexing}

Citation for published version (APA):

Rommel, S., Dodane, D., Grivas, E., Cimoli, B., Bourderionnet, J., Feugnet, G., Morales, A., Pikasis, E., Roeloffzen, C. G. H., Van Dijk, P. W. L., Katsikis, M., Ntontin, K., Kritharidis, D., Spaleniak, I., Mitchell, P., Dubov, M., Barros Carvalho, J., \& Tafur Monroy, I. (2020). Towards a Scaleable 5G Fronthaul: Analog Radioover-Fiber and Space Division Multiplexing. Journal of Lightwave Technology, 38(19), 5412-5422. [9123569]. https://doi.org/10.1109/JLT.2020.3004416

DOI:

10.1109/JLT.2020.3004416

Document status and date:

Published: 01/10/2020

Document Version:

Accepted manuscript including changes made at the peer-review stage

Please check the document version of this publication:

- A submitted manuscript is the version of the article upon submission and before peer-review. There can be important differences between the submitted version and the official published version of record. People interested in the research are advised to contact the author for the final version of the publication, or visit the $\mathrm{DOI}$ to the publisher's website.

- The final author version and the galley proof are versions of the publication after peer review.

- The final published version features the final layout of the paper including the volume, issue and page numbers.

Link to publication

\section{General rights}

Copyright and moral rights for the publications made accessible in the public portal are retained by the authors and/or other copyright owners and it is a condition of accessing publications that users recognise and abide by the legal requirements associated with these rights.

- Users may download and print one copy of any publication from the public portal for the purpose of private study or research.

- You may not further distribute the material or use it for any profit-making activity or commercial gain

- You may freely distribute the URL identifying the publication in the public portal.

If the publication is distributed under the terms of Article $25 \mathrm{fa}$ of the Dutch Copyright Act, indicated by the "Taverne" license above, please follow below link for the End User Agreement:

www.tue.nl/taverne

Take down policy

If you believe that this document breaches copyright please contact us at:

openaccess@tue.nl

providing details and we will investigate your claim. 


\title{
Towards a Scaleable 5G Fronthaul: Analog Radio-over-Fiber and Space Division Multiplexing
}

\author{
Simon Rommel, Member, IEEE, Member, OSA, Delphin Dodane, Evangelos Grivas, Bruno Cimoli, \\ Jerome Bourderionnet, Gilles Feugnet, Alvaro Morales, Evangelos Pikasis, Chris Roeloffzen, Paul van Dijk, \\ Michail Katsikis, Konstantinos Ntontin, Member, IEEE, Dimitrios Kritharidis, Izabela Spaleniak, Paul Mitchell, \\ Mykhaylo Dubov, Member, OSA, Juliana Barros Carvalho, and Idelfonso Tafur Monroy, Senior Member, IEEE
}

(Invited Paper)

\begin{abstract}
The introduction of millimeter wave (mm-wave) frequency bands for cellular communications with significantly larger bandwidths compared to their sub-6 GHz counterparts, the resulting densification of network deployments and the introduction of antenna arrays with beamforming result in major increases in fronthaul capacity required for 5G networks. As a result, a radical re-design of the radio access network is required since traditional fronthaul technologies are not scaleable. In this article the use of analog radio-over-fiber (AROF) is proposed and demonstrated as a viable alternative which, combined with space division multiplexing in the optical distribution network as well as photonic integration of the required transceivers, shows a path to a scaleable fronthaul solution for 5G. The trade-off between digitized and analog fronthaul is discussed and the AROF architecture proposed by blueSPACE is introduced. Two options for the generation of ARoF two-tone signals for mm-wave generation via optical heterodyning are discussed in detail, including designs for the implementation in photonic integrated circuits as well as measurements of their phase noise performance. The proposed photonic integrated circuit designs include the use of both InP and SiN platforms for AROF signal generation and optical beamforming respectively, proposing a joint design that allows for true multi-beam transmission from a single antenna array. Phase noise measurements based on laboratory implementations of ARoF generation based on a MachZehnder modulator with suppressed carrier and with an optical phase-locked loop are presented and the suitability of these transmitters is evaluated though phase noise simulations. Finally, the viability of the proposed AROF fronthaul architecture for the transport of high-bandwidth mm-wave $5 \mathrm{G}$ signals is proven with the successful implementation of a real-time transmission link based on an AROF baseband unit with full real-time processing
\end{abstract}

Manuscript received February 15, 2020; revised April 30, 2020, revised June 17, 2020, accepted June 18, 2020. This work was supported by the blueSPACE project which has received funding from the European Union's Horizon 2020 research and innovation programme under grant agreement No. 762055 .

S. Rommel, B. Cimoli, A. Morales, J. Barros Carvalho and I. Tafur Monroy are with the Institute for Photonic Integration, Eindhoven University of Technology, 5600 MB Eindhoven, The Netherlands (e-mail: s.rommel@tue.nl).

D. Dodane, J. Bourderionnet and G. Feugnet are with Thales Research \& Technology, 1 avenue Augustin Fresnel, 91767 Palaiseau Cedex, France.

E. Grivas and E. Pikasis are with Eulambia Advanced Technologies, 15342 Agia Paraskevi, Athens, Greece.

C. Roeloffzen and P. van Dijk are with LioniX International, $7500 \mathrm{AL}$ Enschede, The Netherlands.

M. Katsikis, K. Ntontin and D. Kritharidis are with Intracom Telecom, Markopoulou Avenue, 19002 Peania, Athens, Greece.

I. Spaleniak, P. Mitchell and M. Dubov are with Optoscribe, Unit 1, Rosebank Technology Park, Livingston EH54 7EJ, United Kingdom.

Color versions of one or more of the figures in this paper are available online at http://ieeexplore.ieee.org.

Digital Object Identifier 10.1109/JLT.2020.XXXXXX of extended $5 \mathrm{G}$ new radio signals with $800 \mathrm{MHz}$ bandwidth, achieving transmission over $10 \mathrm{~km}$ of 7 -core single-mode multicore fiber and $9 \mathrm{~m} \mathrm{~mm}$-wave wireless at $25.5 \mathrm{GHz}$ with bit error rates below the limit for a $7 \%$ overhead hard decision forward error correction.

Index Terms-5G, fronthaul, analog radio-over-fiber, millimeter waves, photonic integration, real-time transmission, phase noise.

\section{INTRODUCTION}

$\mathbf{T}$ HE introduction of fifth generation mobile networks $(5 \mathrm{G})$ is set to drastically expand the range of applications and use cases for mobile communications and to enable massive advancements in the capabilities and performance of mobile networks [1]. More specifically, 5G networks are expected to provide up to $1000 \times$ more capacity, to support a significantly larger number of users per area, to reach multi-Gbit/s end user data rates and to support latencies down to $1 \mathrm{~ms}$ - all to support applications ranging from media consumption and broadcasting on-the-go to autonomous driving and the control of robots, production plants and entire factories [2]. In order to fulfill such expectations, $5 \mathrm{G}$ networks introduce major changes to the radio access network (RAN) in terms of architecture, degrees of centralization and placement of processing functionality, in order to ensure the required data rates, quality of service (QOS) and reduced latency [3], [4]. In this context, the introduction of novel strategies and technologies becomes a key factor [5], [6], including the introduction of millimeter wave (mm-wave) radio frequencies [7], [8].

The latter is considered key to achieve the required end user data rates as at mm-wave significantly more spectrum is available for mobile networks compared to the overcrowded region of traditional mobile communications frequency bands sub- $6 \mathrm{GHz}$ and despite the introduction of additional spectrum in the latter. While the availability of additional spectrum allows larger over-the-air capacities, the introduction of highbandwidth mm-wave signals brings significant challenges as the viable range of signals - and thus size of the mm-wave cells - will typically be below $500 \mathrm{~m}$, requiring a significantly more dense network deployment [9], [10]. This network densification, together with the use of larger bandwidths and largescale multiple-input multiple-output (MIMO) or beamforming, further requires a major upgrade or re-design of centralized radio access networks (C-RANs), as current solutions do not 
scale well especially in the fronthaul segment, i.e., between the radio equipment or remote unit (RU) and the baseband unit (BBU) at the central office (CO) [3], [4].

Analog radio-over-fiber (AROF) was initially suggested for fixed wireless access or wireless bridges between fiber networks [11], [12] and includes both the pure analog transport of radio frequency (RF) signals and the use of microwave photonics for optical signal processing or optical heterodyning for frequency conversion [13]. More recently, AROF has received significant interest for $5 \mathrm{G}$ fronthaul as a solution to solve the fronthaul capacity issues and through optical heterodyning assist in mm-wave generation with maximum centralization of network resources [4], [14], [15]. ARoF at the same time leads to new considerations for the RAN, as it must be taken into account in resource allocation and network control [16] and because it effectively merges the fiber optical and radio channel and their associated impairments [17]. With optical heterodyning, phase noise in particular may be a relevant limitation and must be tightly controlled when designing and implementing AROF transmission schemes [18], [19].

While ARoF fronthaul holds a lot of promise for mm-wave $5 \mathrm{G}$ networks, the realization of such promise will strongly depend on the use of photonic integration and the implementation of AROF transceivers in photonic integrated circuits (PICs). Only with such integration can ARoF systems achieve the spatial and energy footprints required for ubiquitous and densely deployed $5 \mathrm{G}$ mm-wave with ARoF fronthaul and also be attractive from a cost perspective [20]. Integrated ARoF transceivers further enable the use of optical beamforming, where analog beamforming functionality for mm-wave signals is implemented in compact microwave photonic circuits rather than bulky and power hungry RF electronics [21]. PICs for microwave frequencies and AROF transmitters have been under study, however successful implementations of AROF transceivers in their full complexity are yet to emerge.

Beyond the introduction of AROF with PIC-based transceivers, optical space division multiplexing (SDM) can provide a significant capacity upgrade in the RAN and further allows sharing of a common passive optical distribution network (ODN) for both radio access and other fiber-based access networks, such as passive optical networks (PONs) [22]. While initially suggested as a method to push the ultimate capacity of fiber networks using spatial multiplexing in different cores or modes or a combination thereof [23], the use of SDM with multi-core fiber (MCF) in RANs has gained significant interest, especially in connection with AROF transport and the use of optical beamforming [21], [24], [25].

The combination of AROF and optical beamforming in PICs with MCF for AROF transport forms the central technology proposition for $5 \mathrm{G}$ fronthaul for high-bandwidth mm-wave signals of the EU H2020 5G-PPP project blueSPACE [26] and was the topic of two workshop contributions at MWP 2019 by the authors, serving as the basis for this manuscript. This manuscript expands upon these, briefly discussing the AROF fronthaul over SDM scheme of blueSPACE and introducing in some detail its ARoF transmitter schemes and designs for their implementation in integrated photonics. In preparation towards a full implementation, it provides experimental phase noise measurements for two different mm-wave ARoF transmitter schemes and present simulations for phase noise requirements and tolerances in systems with $5 \mathrm{G}$ new radio (NR) orthogonal frequency division multiplexing (OFDM) signals and real-time processing in an AROF BBU. Finally, real-time transmission of $800 \mathrm{MHz}$ wide extended $5 \mathrm{G}$ NR signals is successfully demonstrated for an ARoF fronthaul link with 7-core MCF and a $9 \mathrm{~m} \mathrm{~mm}$-wave link at $26.5 \mathrm{GHz}$ [16], [27], i.e., in the 3GPP n258 band [28]. The demonstration is based on an ARoF BBU implemented on an field programmable gate array (FPGA) and intermediate frequency (IF) AROF transport with optical heterodyning for mm-wave upconversion of the downlink signal and features a remote-fed RF local oscillator (LO) for electrical downconversion of the uplink signal, maximizing centralization of network equipment at the $\mathrm{CO}$ and minimizing RU complexity. Together these contributions show the viability of the blueSPACE architecture and establish performance requirements for the realization of the AROF transmitters and optical beamforming networks (OBFNs) in PICs.

The remainder of the manuscript is structured as follows: section II discusses ARoF fronthaul systems for mm-wave $5 \mathrm{G}$ (II-A), introduces the relevant ARoF transmitter schemes (II-B) and considers the possibilities for their integration in PICs (II-C). Section III introduces the blueSPACE ARoF fronthaul architecture with optical beamforming (III-A) and corresponding ARoF transmitter PIC designs (III-B). Section IV discusses phase noise in OFDM-based mm-wave ARoF systems, first, showing the impact of phase noise on the OFDM transmission performance and, second, presenting measurements of phase noise from two ARoF transmitter schemes. Section V presents the experimental transmission of extended 5G NR signals with ARoF fronthaul over SDM and mm-wave RF transmission. Finally, section VI summarizes and concludes the paper.

\section{Analog Radio-over-Fiber Fronthaul Systems FOR MILLIMETER-WAVE 5G}

This section discusses the advantages of moving from digitized to analog transport in the fronthaul link and introduces a basic architecture for analog fronthaul, before discussing different ARoF transmission schemes and the possibilities for their implementation in photonic integrated circuits.

\section{A. Centralized Radio Access Networks with Analog Radio- over-Fiber}

The move from distributed radio access networks (D-RANs) with baseband processing performed at every remote site to centralized radio access networks (C-RANs) with a shared and centralized baseband unit (BBU) pool has proven successful in reducing the cost of network ownership, operation and maintenance [29]. By centralizing all baseband processing in a BBU pool at the central office (CO), as shown in Fig. 1(a) and (b), C-RAN allows statistical multiplexing and sharing of baseband processing resources and simplifies the management and maintenance of the network by reducing the complexity placed near the antennas at remote locations. At the same time however, the introduction of C-RAN introduced a new segment 


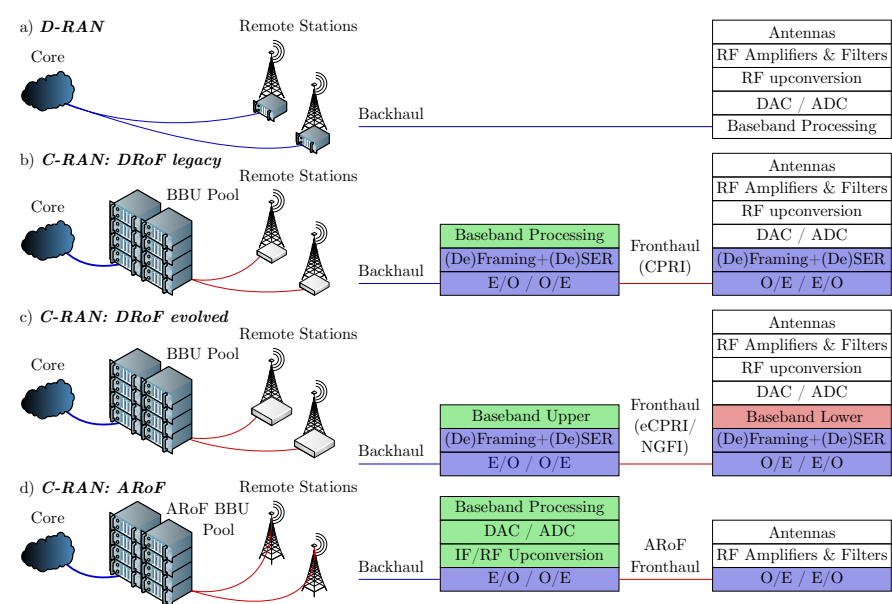

Fig. 1. Comparison of RAN options and corresponding placement of signal processing functions: a) D-RAN with full baseband processing at the remote site, b) traditional C-RAN with DROF CPRI fronthaul and centralized baseband processing, c) evolved C-RAN with DROF eCPRI or NGFI fronthaul and functional split, d) C-RAN with ARoF fronthaul and full centralization of baseband processing and RF sources. SER: seralization, O/E \& E/O: optical to electrical and electrical to optical conversion.

in the RAN, the fronthaul segment between BBU at the $\mathrm{CO}$ and remote unit (RU) at the antenna site.

In pre-5G networks, this segment was covered by CPRI based fronthaul - i.e., transmitting the digitized IQ samples of the target or received OFDM waveforms - typically over fiber optic links and hence referred to as DRoF. The transmission of digitized samples however requires constant bitrate signals, where the rate scales with signal bandwidth and antenna configuration and is independent of user presence or activity [3]. Furthermore, with multi-sector or even MIMO antennas, these fronthaul links scale to hundreds of Gbit/s and into the Tbit/s even for moderate signal bandwidths and MIMO configurations and thus, together with their stringent requirements on latency and jitter, are clearly not a scaleable solution for high-bandwidth 5G signals, especially at mm-wave.

One approach to mitigate the resulting fronthaul capacity bottleneck is the use of DRoF with eCPRI or NGFI, introducing a partial re-distribution of the baseband processing and a functional split, as shown in Fig. 1(c) [30]. While this can significantly reduce the required data rates and - depending on the functional split chosen - may reduce latency and jitter requirements [30], it requires the introduction of an additional distributed unit (DU) between the CO and RU and thus results in a partial loss of the centralization gain.

AROF on the other hand solves both the fronthaul capacity problem and maintains the centralization gain by moving from the transport of digitized radio samples to the analog transport of the baseband, IF or RF waveforms. By moving to analog transport, not only does AROF fronthaul maintain the centralization of the complete baseband processing, it further centralizes the digital to analog converter (DAC) and analog to digital converter (ADC) stages otherwise located at the RU, as shown in Fig. 1(d). The optical bandwidth or spectrum required for AROF transport directly relates to the IF or RF frequency transported or targeted plus the signal bandwidth

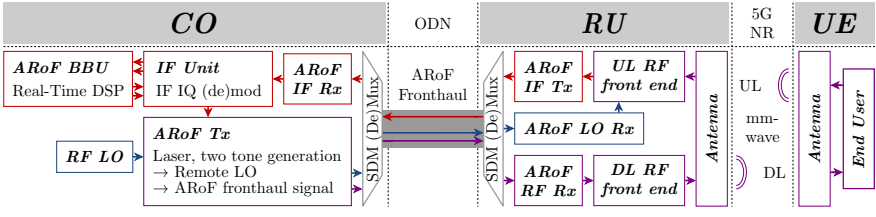

Fig. 2. General ARoF fronthaul architecture with optical heterodyne upconversion for downlink, a remote-fed LO for downconversion of the uplink signal at the RU before IF ARoF transport to the CO.

and - with narrow optical filtering or multiplexing - may even be reduced below that by interleaving optical carriers and modulation sidebands with narrow channel spacings [15], [22]. If combined with optical heterodyning, ARoF becomes especially interesting for mm-wave $5 \mathrm{G}$ NR signal generation as the ARoF link can not only transparently transport an IF signal, but also perform its upconversion to $\mathrm{mm}$-wave through optical heterodyning on the photodiode (PD) at the RU - while keeping the required RF oscillator at the CO [14], [27].

A corresponding ARoF fronthaul architecture is shown in Fig. 2, using IF AROF transport for the downlink and intermediate frequency-over-fiber (IFOF) transport for the uplink [15], [31]. It features the AROF BBU and IF unit at the $\mathrm{CO}$, as well as an AROF transmitter and IFOF receiver. The ARoF transmitter generates both the ARoF signal for optical heterodyning as well as a two-tone signal used as remote-fed LO for electrical downconversion at the RU. The latter includes an AROF receiver, downlink RF front end and the antenna in downlink direction, before radiation of the mm-wave signal over the 5G NR air interface to the user equipment (UE). In uplink direction the signal received from the UE passes the uplink RF frontend, including downconversion to IF using the remote-fed LO, before being transmitted as an IFOF signal back to the $\mathrm{CO}$. With this basic architecture, no RF source is required at the RU, while employing IFoF transport for the uplink to reduce the required bandwidth of the driver amplifier (DA) and optical modulator.

The use of mm-wave bands further results in a significant reduction in antenna size and brings the possibility to use phased array antennas (PAAs) or other multi-element antenna systems, either for massive MIMO or with analog beamforming or a hybrid solution of both [8]. The basic AROF link is easily expanded to use analog optical rather than electrical beamforming to further reduce spatial, power and cost footprints of the RU. The implementation of such an ARoF solution is, however, not trivial and a number of options exist for the AROF transport and the generation of the two-tone signal required for optical heterodyning, which are introduced in the following. The AROF fronthaul architecture with optical beamforming and MCF transport proposed by blueSPACE is discussed in section III-A with details on the integrated AROF transmitter designs given in section III-B.

\section{B. Analog Radio-over-Fiber Transmitter Schemes}

Analog fronthaul over optical fiber can be achieved either by directly modulating the optical carrier with the RF signal or by modulating it with an IF or baseband signal and subsequent 


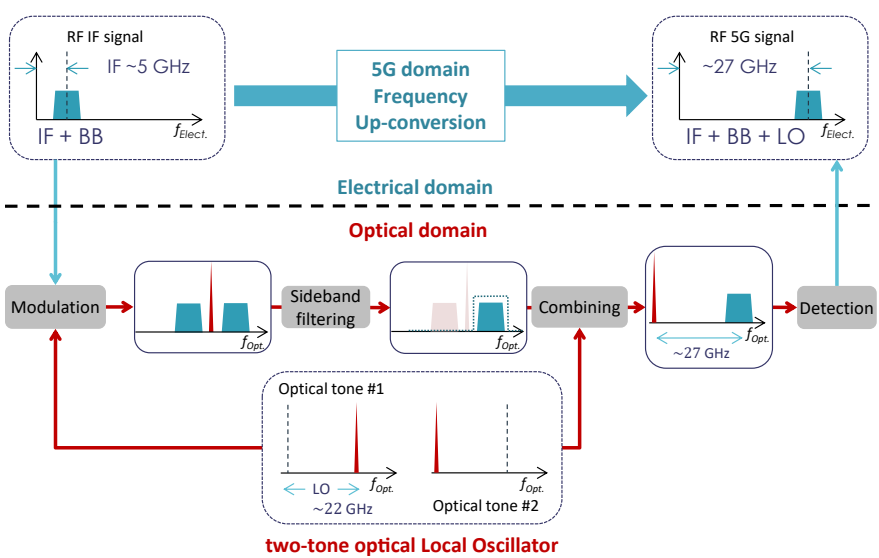

Fig. 3. Description of the frequency upconversion from modulated IF to mm-wave RF using optically generated two-tone signals.

upconversion at the remote side in the electrical domain or through optical heterodyning [13], [15], [31]. While for traditional radio frequencies direct modulation with the RF signal is feasible, for mm-wave signals this is limited by modulator bandwidth and schemes based on optical heterodyning are preferred as the use of optical carriers for $5 \mathrm{G}$ $\mathrm{mm}$-wave signal generation provides significant advantages. First, it is relatively simple to generate optical signals that are separated by an offset frequency in the range of the $5 \mathrm{G}$ mm-wave spectrum $(\approx 30 \mathrm{GHz})$ and beyond and the tunability of telecom laser sources makes the full frequency range very accessible. Second, the modulation bandwidth available from optical components facilitates the use of larger signal bandwidth as available at mm-wave. These aspects of microwave photonics are crucial advantages of AROF for $5 \mathrm{G}$ applications.

The goal for an ARoF transmitter is to generate the modulated mm-wave 5G RF carrier by means of optical elements, i.e, the electrical to optical (E/O) conversion of the data carrying waveform and its upconversion to mm-wave. A general description of the upconversion of an IF signal to mm-wave frequencies is shown in Fig. 3. First, the IF signal modulates an optical carrier, creating sidebands in the optical spectrum of the signal. The signal is then filtered so that only one sideband remains and is combined with another optical signal (generally referred to as LO). Finally, a fast PD performs the optical to electrical $(\mathrm{O} / \mathrm{E})$ conversion and through optical heterodyning generates an RF signal at a frequency equal to the sum of the difference between the two optical tones and the IF. The key aspect of this E/O/E link is the generation of the two optical tones shown at the bottom of Fig. 3, as it has a major impact on the useability of the two-tone signal and the overall transmission performance.

The main criteria to evaluate the optical two-tone generation are the tunability, which reflects the flexibility of the system to cover the full $5 \mathrm{G}$ frequency range, the power budget, which describes its power efficiency, and the resulting signal purity. Several solutions exist for two-tone generation, generally involving either several laser sources, each corresponding to one tone, or one single laser source, used to generate multiple tones. The former is more power efficient but suffers from coherence issues while mixing signals which originate from different laser sources. The latter is very commonly used to avoid coherence issues between lasers that impact signal purity, but at the cost of a limited power budget due to having only one optical power source.

On one hand, solutions involving a single laser source are mainly based on the use of interferometric modulators and/or optical resonators. In both cases multiple optical tones are generated from one original carrier which means that their heterodyne recombination will not suffer from coherence issues, given temporal alignment of the tones is maintained during processing. This can be done using carrier-suppressed Mach-Zehnder modulators (SC-MZMs), which is a wide-spread method to generate such a two-tone optical signal and to perform effective frequency doubling [13], [31], [32]. The main limitation of this solution is linked to power efficiency as a significant portion of the input optical power is lost due to the suppression of the carrier. In order to compensate for this, amplifier stages (e.g., semiconductor optical amplifiers (SOAs) or erbium-doped fiber amplifiers (EDFAs)) are typically required, decreasing the overall efficiency. Similarly, power efficiency when using mode-locked laserss (MLLs) or optical frequency combs [33], [34] is also critical as the optical power is transferred into multiple tones while only a few are used at any time, with filtering often required to get rid of unused comb frequencies.

On the other hand, solutions including separate lasers are more power efficient since power is not spread from one to multiple tones, but suffer from coherence issues inherent to the use of different sources. In particular, the pure heterodyne beating of two semiconductor lasers (SLs) is very simple to perform and strongly benefits from their tunability. Yet, integrated SLs generally have rather high intrinsic levels of phase noise, which can substantially deteriorate system performance or even make impossible to achieve signal purity requirements set in relevant standardization and regulations [19]. As a result, the use of independent lasers is only feasible if the intrinsic laser phase noise becomes very small.

An alternative solution is to make the laser sources "artificially" coherent through the use of an electronic external control loop. This method is referred to as optical phaselocked loop (OPLL) and its implementation in PICs has already been demonstrated [35]-[37]. The final beating is purely heterodyne, but the two lasers are strongly correlated to the point of appearing to be identical, resulting in a potentially very pure RF tone. This type of architecture can be very useful as it is possible to use as many lasers as desired optical tones [38], allowing the full power of one laser in each tone and consequently avoiding optical amplifiers. It is worth noticing that electrical PLLs have become very common components, in particular for demodulation of signals and frequency upconversion of low-jitter clocks [39]. However their optical equivalents are critical to design and can prove to be quite difficult to implement in a functional device. They further have finite bandwidth, meaning that the two lasers have correlated phase noises only within a certain frequency range around their central frequency. 


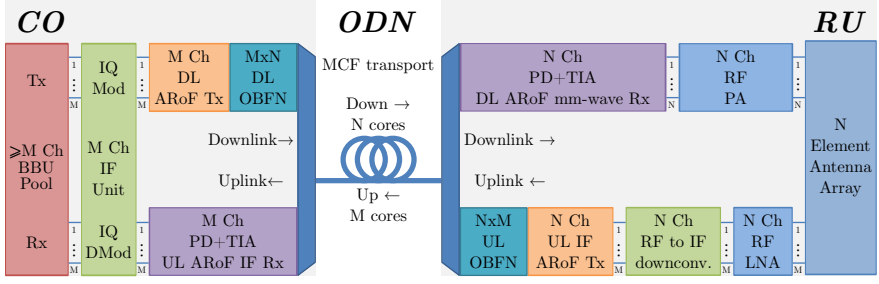

Fig. 4. blueSPACE architecture with optical beamforming and ARoF transport over MCF: the downlink OBFN is centralized and MCF transport is employed to maintain signal synchronization during transport; the uplink is downconverted electrically and optical beamforming for the IF signal takes place at the RU.

\section{Photonic Integration of ARoF Transmitters}

While it is clear that AROF is key to the realization of future $5 \mathrm{G}$ networks, it is obvious that its full potential can only be exploited through a strong scalability and optimized power efficiency while minimizing cost and footprint. This is where integrated photonics becomes a real asset and the latter is especially true when speaking of optical beamforming integrated devices, which have proven to be a very promising field of research for aerospace, radar and telecom applications in the recent years [21], [40]. Several architectures and designs of integrated circuits have been developed in order to exploit PICs to support the growing interest [41], and in particular for the realization of OBFNs in silicon nitride (SiN) [42], [43]. The implementation of an OBFN within an integrated transmitter requires first to generate a proper optical signal on which the IF will be modulated as well as an optical LO. In this section we discuss the available choices in terms of PICs to perform this step and their compatibility with an SiN OBFN.

The choice of a foundry or a photonics platform for PICs fabrication is actually strongly determined by the choice of a method to generate the optical IF and LO tones. Given commercially available possibilities a fully-integrated solution would necessarily include monolithically integrated laser sources, which is only available through indium phosphite (InP) foundries [44]. Yet, if no integrated source is used then a fully silicon-based approach can be developed, including the OBFN, with an external laser source. The main advantage of such a scheme relies eventually on the use of a very pure and powerful laser source combined with silicon photonics great performances in terms of footprint and optical losses. This approach is suitable for IF modulation and circuitry and benefits from a strong compatibility with sin, which is particularly popular for complex circuits and optical beamformers [42], [43], [45]. Thus, it is worth mentioning that significant progress has been made in the field of hybrid integration of III-V materials on silicon wafers [46], which could prove to be worth in the future as a solution for fully-integrated active/passive devices.

However, such technologies are not state of the art and are not well-suited for a fabless approach and the current best choice in terms of scalability is to rely on InP foundries [44] for the generation of optical IF and LO signals. These platforms can provide multi-project wafer (MPW) runs of standardized PICs in a commercial manner, fitting a fabless approach, and can deliver all the necessary components for $5 \mathrm{G}$ applications such as high-speed modulators and photodiodes, as well as optical amplifiers. Furthermore, this process is compatible with coupling to an SiN chip for the OBFN, as has already been demonstrated for other high performance devices [47]. Design considerations for InP PICs are presented in the next section where we propose a system architecture including AROF as well as an implementation of two different methods to generate optical tones for mm-wave $5 \mathrm{G}$ applications: single on-chip laser with SC-MZMs and an external OPLL.

\section{BLUESPACE ANALOG RADIO-OVER-FIBER ARCHITECTURE AND INTEGRATED TRANSMITTERS}

\section{A. ARoF Fronthaul Architecture with Optical Beamforming}

The blueSPACE fronthaul architecture, shown in Fig. 4, expands the basic AROF architecture previously shown in Fig. 2 through the use of optical beamforming and optical space division multiplexing in MCF. At the $\mathrm{CO}$, the architecture features a pool of AROF BBUs and IF units for baseband signal processing and IF up-/downconersion. The downlink transport is implemented with an IF ARoF link with optical heterydyning, composed of an integrated multi-channel AROF transmitter and an OBFN at the $\mathrm{CO}$, transport of the beamformed signals over a MCF and an AROF receiver with $\mathrm{PD}$ and transimpedance amplifier (TIA) for mm-wave generation and amplification. Further amplification is provided by a set of power amplifiers (PAs) before radiation from the PAA. The received uplink signal is amplified with a bank of low-noise amplifiers (LNAs) and downconverted to IF, before entering an IFoF link with optical beamforming at the RU and MCF transport to the IFOF receiver with PD and TIA at the CO.

The optical beamformer can be implemented either as a coherent optical beamformer based on an optical implementation of a Blass matrix [48] or an incoherent beamformer with truetime delays. In either case, optical beamformers can achieve true multi-beam transmission from a single antenna array, effectively forming a mapping matrix between $M$ beam inputs and $N$ outputs towards the $N$ antenna elements (in downlink direction) in which each input is mapped to each output with a variable, progressively increasing delay or phase shift. In uplink direction, vice versa, $N$ inputs from the $N$ antenna elements are copied to all outputs, but with correctly adjusted delays to spatially filter one beam direction towards each of the $M$ outputs. It should be noted that, in order to achieve non-overlapping beams, naturally $N>M$, i.e., the number of antenna elements is larger than the number of beams produced, and thus the beamforming matrix is asymmetric.

With the introduction of optical beamforming comes the question of placement of the downlink OBFN, as having the OBFN at the CO (as shown in Fig. 4) maximizes centralization, but poses stringent requirements on the fronthaul link, while placing it at the RU comes with relaxed requirements at the cost of increased RU complexity and power consumption. The differences in requirements are a direct result of the OBFN function of mapping $M$ input signals for $M$ independent beams to $N$ combined output signals with well defined phase relations that define the shape and direction of the resulting beams. Hence, with the OBFN at the $\mathrm{CO}$, the fronthaul link 


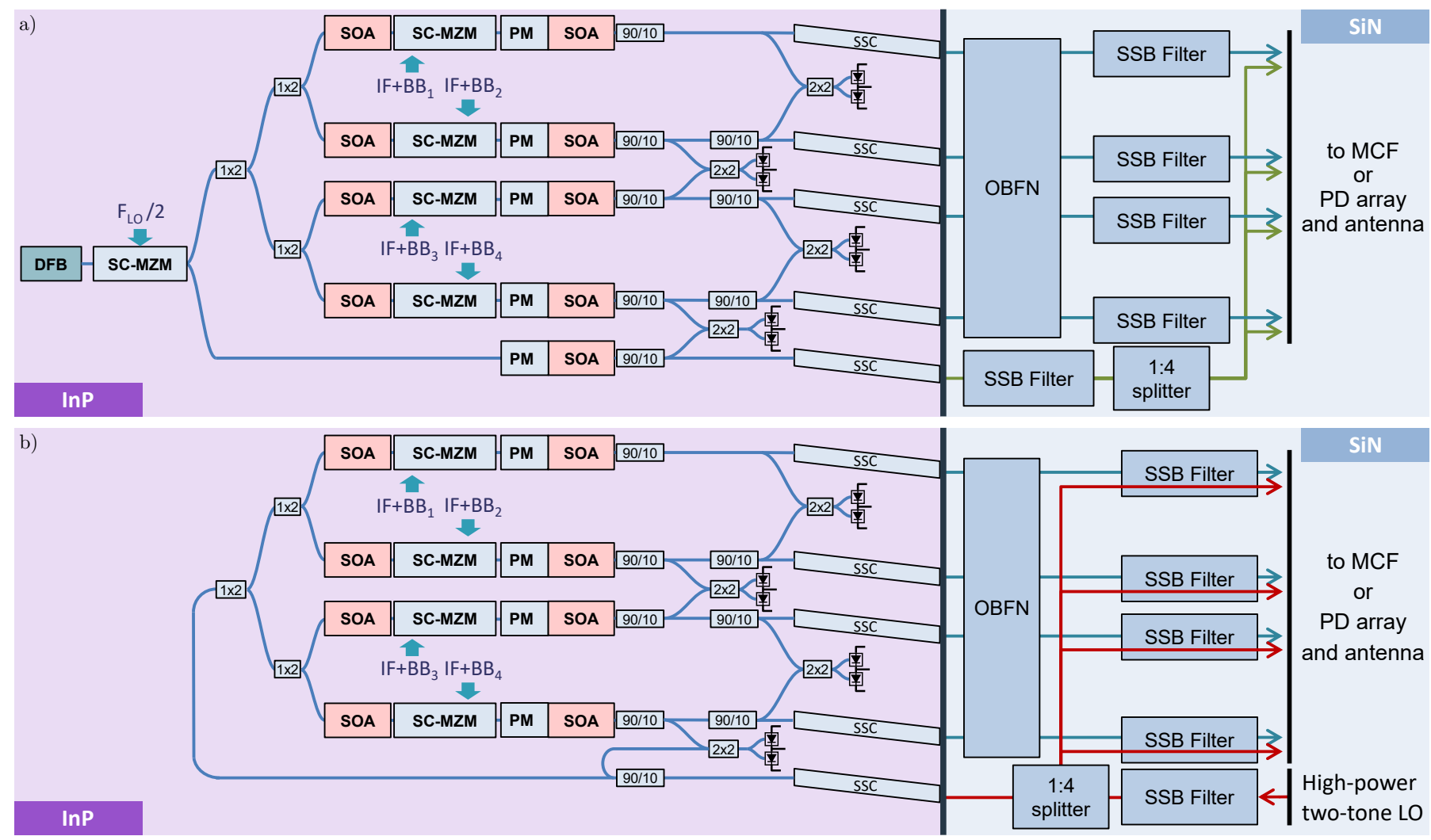

Fig. 5. Architecture of an AROF integrated transmitter with: a) a single on-chip laser and a SC-MZM for LO generation, b) an external OPLL providing a high power two-tone LO. SSC: spot size converter.

must transport $N$ parallel signals and maintain their temporal synchronization within a narrow margin to preserve the beam patterns established by the OBFN. The use of MCF for transport of the fronthaul signals is key with regards to this aspect, as it allows parallel transport of multiple signals at the same wavelength and is expected to have significantly smaller differential delays between channels than solutions based on single-mode fiber (SMF) ribbons or bundles [49]. With the OBFN at the RU on the other hand, the number of parallel signals corresponds to the number of beams $M$ rather than the number of antenna elements and, as the transported signals are a priori independent, temporal synchronization is not required. A similar discussion applies in uplink direction, where placement of the OBFN at the RU again reduces the synchronization and scaling requirements compared to having the OBFN centralized at the CO.

\section{B. blueSPACE Integrated ARoF Transmitter Designs}

In this section we present two designs illustrating how the two of the aforementioned AROF transmitter solutions are implemented in practical PICs, designed to directly support optical beamforming and multi-beam transmission. In the interest of scalability, we focus on using only commercially available technologies which we use to design our own circuits. The main goal of this approach is to demonstrate the feasibility of such an architecture with readily available technology. The functions of the E/O/E ARoF link of Fig. 3 can be grouped as follows: optical generation and E/O conversion of the signal, implemented in InP, optical beamforming, implemented in SiN, and photodetection, again implemented in InP. The first two steps are referred to as AROF transmitter and the last one as AROF receiver. Transmitter and receiver can be separated by optical fiber to allow transmission of the AROF signal over long distances. Designs of two ARoF transmitters based on the methods described in section II-B are presented in Fig. 5.

The first, shown in Fig. 5(a), uses a single on-chip distributed feedback laser (DFB) followed by a SC-MZM to generate a $22 \mathrm{GHz}$ two-tone optical LO signal. This signal is separated into an LO path and an IF modulation path. As both paths contain the two optical tones, sideband filtering is required further in the architecture. The IF modulation path is then split into four channels, each being modulated with a different IF signal at $\approx 5 \mathrm{GHz}$, allowing for the simultaneous generation of four AROF signals from a single transmitter. A set of balanced PD and carrier-injection phase modulators (PMs) allows controlling the residual phase differences between channels before the light is coupled out of InP chip. The assembly of the InP AROF transmitter and SiN OBFN chip is made by butt-coupling and mounted on a dedicated printed circuit board ( $\mathrm{PCB})$. In the sin chip one of the LO tones is filtered out, while, in parallel, the four IF modulated channels are routed through the OBFN. Then the opposite sideband compared to the LO is filtered from the beamformed modulated signals. Finally, each of the four modulated channels is recombined with a copy of the now single-tone LO. The resulting signals contain all processing 


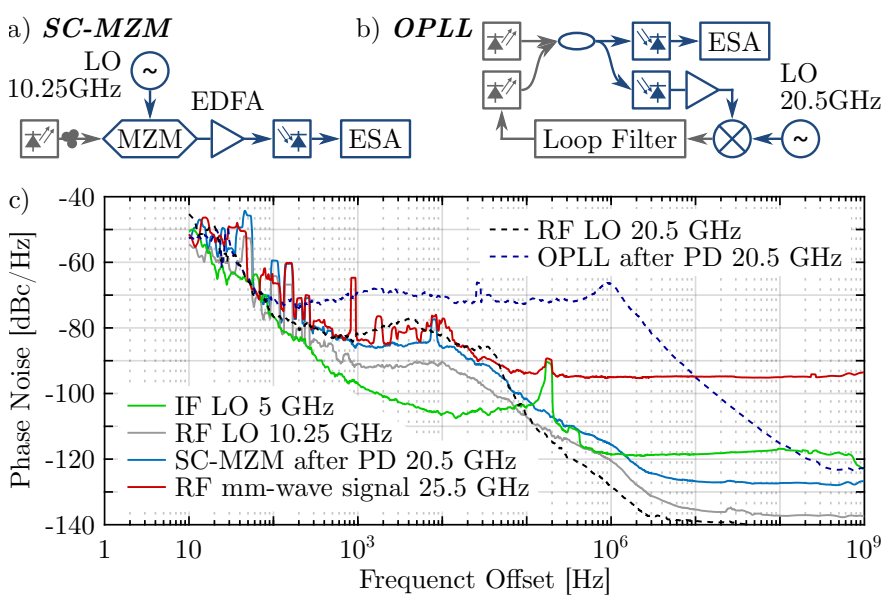

Fig. 6. Phase noise measurements for two-tone generation with SC-MZM and OPLL: a) measurement setup with SC-MZM, b) measurement setup with OPLL, c) comparison of phase noise for the two-tone generation methods and phase noise of the overall mm-wave signal of the ARoF fronthaul link (see section V).

required for radiation by the PAA and can be sent either directly to a PD array or through a MCF to the RU.

The main advantage of this scheme is that it uses a single laser, avoiding coherence issues between optical signals. However, its limitation is a restricted power budget, as onchip lasers are currently limited to a few $\mathrm{dBm}$ optical power. In order to compensate for this, SOA arrays are used to reamplify signals before IF modulation and out-of-chip coupling. Nonetheless, this limits the achievable MCF transport distance to the RU. In particular the one to four splitting and single sideband (SSB) filtering of the two-tone LO being performed after the last SOA severely impact power budget. However this design is highly flexible and can be used directly with a PD array to form an E/O/E beamforming block that can be incorporated within any RF link.

The second option, shown in Fig. 5(b), is designed to use high power LO tones to provide a better power budget of the $\mathrm{E} / \mathrm{O} / \mathrm{E}$ link. As it is difficult to achieve high optical powers with commercially available monolithically integrated InP lasers, the optical carrier is generated off-chip with an external OPLL or optical fiber SC-MZM. This also offers the advantage of allowing the high power laser units to be centralized at the CO even if the OBFN is placed at the RU. The use of an off-chip two-tone generation requires some changes compared to Fig. 5(a): the external two-tone signal is first fed through the sin chip where the tones can be easily separated using ring resonator filters. One of the tones is then coupled into the InP chip where the IF modulation is performed using the same Mach-Zehnder modulator (MZM) tree as in the single laser architecture. The OBFN and final sideband filtering are also identical to the first option. However, by only feeding a single carrier to the IF modulation stage, the use of the SOAs is significantly more efficient, as their gain is not shared by multiple, unused carriers. After combination with the other tone at high power, the processed signal can be transmitted over multiple $\mathrm{km}$ through MCF. Another advantage of this architecture is that sin chips can handle more optical power thus allowing to use a high power LO without suffering from InP chip limitations.

In order to feed this transmitter design with a two-tone optical LO, a dual-output OPLL setup has been implemented, delivering a signal with up to $40 \mathrm{~mW}(16 \mathrm{dBm})$ optical power per tone and two optical outputs $(160 \mathrm{~mW}$ useful power in total without needing any additional amplifier). While twotone generation with a SC-MZM provides sufficient phase noise performance for AROF systems, this is not necessarily the case with an OPLL, especially when real-time processing is required. In order to ensure that the final implementation is viable, a key step is to investigate the influence of the phase noise of such an OPLL on real-time data transmission. In section IV simulations are presented to estimate the required phase noise performance and validate the use of an OPLL in the proposed architecture, which would include the PIC described in this section.

\section{Phase Noise in OFDM based Millmeter-WaVe ANALOG RADIO-OVER-FIBER SYSTEMS}

Phase noise plays a fundamental role in the performance of radio communication systems and limiting phase noise is one of the major concerns to be addressed before the introduction of AROF links in fronthaul networks. While phase noise in AROF systems has been studied [50]-[52], its impact on real-time processing of AROF OFDM signals remains to be evaluated. Consequently, in this section the measured phase noise performance of two of the aforementioned two-tone generation schemes is discussed and simulations are presented aiming at determining the maximum acceptable level of phase noise for real-time processing with the AROF BBU.

\section{A. Phase Noise Measurements}

The experimental evaluation of phase noise resulting from two-tone generation as required for an AROF fronthaul focuses on the two schemes for two-tone generation discussed above, namely the SC-MZM and OPLL approaches. Both are implemented with bulk optical components and their respective setups are shown in Fig. 6(a) and (b). Both signals were set to have approximately similar power RF levels after heterodyning on the PD. Phase noise is directly measured using an electrical spectrum analyzer (ESA), without amplification after the PD.

The resulting phase noise power across offsets from the carrier between $10 \mathrm{~Hz}$ and $1 \mathrm{GHz}$ is shown in Fig. 6(c), alongside reference measurements of the RF LOs employed. The phase noise profile with the SC-MZM follows that of the underlying RF LO, but increased by about $6 \mathrm{~dB}$, as expected due to the effective frequency doubling. It features a small flat area at $-85 \mathrm{dBc} / \mathrm{Hz}$ from $0.8 \mathrm{kHz}$ to $15 \mathrm{kHz}$ and then slopes down to below $-120 \mathrm{dBc} / \mathrm{Hz}$ at $2 \mathrm{MHz}$ offset from the carrier. In comparison, the phase noise measured for the OPLL setup shows a significantly different behavior. At low frequencies the OPLL phase noise profile approximately matches that of the underlying RF LO, then remains flat at a level around $-70 \mathrm{dBc} / \mathrm{Hz}$ until a "shoulder frequency" of around $1 \mathrm{MHz}$, beyond which it slopes down towards $-120 \mathrm{dBc} / \mathrm{Hz}$ at $200 \mathrm{MHz}$ offset from the carrier. This "shoulder frequency" corresponds 
to the bandwidth of the OPLL, beyond which the phase noise profile returns to that of the lasers if left free-running. While this confirms the correct operation of the OPLL, it does raise the question of whether such levels of phase noise can be handled by a real-time AROF transmission system and by how much performance would be degraded - this is evaluated in the simulations provided in the next section.

Finally, as a reference, Fig. 6(c) further includes the phase noise measured for the IF carrier generated by the IF unit and the final $25.5 \mathrm{GHz}$ mm-wave RF signal after modulation of the SC-MZM two-tone signal with the IF and optical heterodyning for upconversion (as performed in the experimental fronthaul link demonstration discussed in section V). As can be seen, the final mm-wave signal contains phase noise contributions from both two-tone and IF signals, but overall stays well below the phase noise level observed for the locked lasers. It should be noted that the different levels at which the sensitivity floor appears in the graphs is entirely due to the different power levels of the analyzed signals.

\section{B. Phase Noise Simulations}

As phase noise is one of the main differentiating parameters between the two-tone generation setups, a set of OFDM simulations was conducted to study the impact of phase noise on the AROF BBU and to estimate the required improvement in phase noise for the OPLL to become a feasible alternative. These simulations included a reduced version of the experimental AROF link that comprises an ARoF BBU in a loopback configuration at the intermediate frequency, as the scope was to get an insight into the AROF BBU real-time digital signal processing (DSP) performance in the presence of phase noise, rather than to simulate the overall link. In this configuration, phase noise - shaped to match the experimental measurements of the OPLL - was inserted in the stages of up-/downconversion to/from the intermediate frequency from/to baseband signals.

Bit accurate models of the main functional blocks of the original ARoF BBU implemented in the context of blueSPACE were developed in Matlab and used for the simulation results presented in this section, in order to better understand the tolerance of the real-time hardware implementation to phase noise. Specifically, bit-accurate models of the fast Fourier transform (FFT) engines and the complete receiver DSP chain, including the synchronization stage, the carrier frequency offset compensation stage and the zero-forcing equalization stages were developed and used in these simulations. The signals employed are extended 5G NR OFDM signals with subcarriers spaced at $240 \mathrm{kHz}$ and a bandwidth of $800 \mathrm{MHz}$.

The different phase noise profiles used in the simulations are shown in Fig. 7(a), alongside the measured phase noise of the RF carrier generated by the OPLL when set at half its maximum optical output power. Several phase noise profiles have been designed to represent the behavior of an ideal OPLL RF carrier with a phase profile that is flat up to a given frequency and then follows a $1 / f^{2}$ slope corresponding to two lasers in free-running mode. The range of the simulated levels of the initial flat part of the phase profile is between $-75 \mathrm{dBc} / \mathrm{Hz}$ and $-115 \mathrm{dBc} / \mathrm{Hz}$, ranging from a level similar
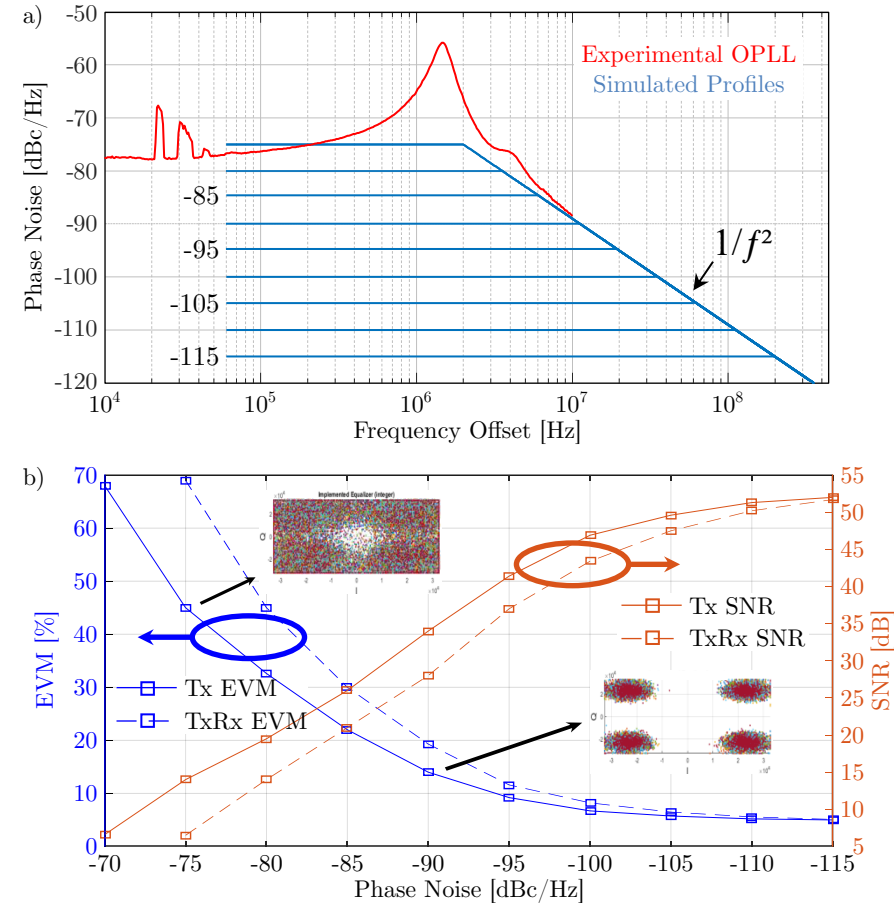

Fig. 7. Simulation results of the influence of phase noise on the OFDM transmission: a) phase noise profiles used as input of the simulation and measured OPLL phase noise, b) corresponding simulated OFDM EVM and SNR as a function of phase noise level for transmission (Tx) and transmission and reception (TxRx); insets show two of the corresponding constellations.

to that observed from the OPLL to below that observed with the MZM two-tone generation. It should be noted that this approximation does not reflect the fact that for low phase noise levels both methods are limited by the phase noise of the RF source. By maintaining the horizontal position of the slope, it is also implicit that the lasers in the hypothetical OPLLs with lower phase noise remain the same, and thus a physical equivalent of these simulations would correspond to an improvement of the overall OPLL performance, for instance by improving the loop filter. The simulations do not take into account the satellite peak at the shoulder frequency generated by the feedback loop.

The results of the simulations are presented in Fig 7(b), where the EVM and SNR of the transmitter only and the transmitter and receiver combined are plotted as a function of the phase noise level. One can see that for the real OPLL setup $(-70 \mathrm{dBc} / \mathrm{Hz}$ to $-75 \mathrm{dBc} / \mathrm{Hz})$, the EVM stays above $40 \%$ which leads to a heavily degraded signal constellation. The SNR of the measured output signal is around $10 \mathrm{~dB}$ in that case. It can further be observed that, as the flat phase noise level reduces, EVM reduces and it is possible to reach EVM values below $10 \%$ as well as SNRs up to $50 \mathrm{~dB}$. These simulations suggest, that reasonable EVM and SNR values can be reached with an OPLL noise level of $-90 \mathrm{dBc} / \mathrm{Hz}$, i.e., around $15 \mathrm{dBc} / \mathrm{Hz}$ below the initial real OPLL setup.

As evident from the experimental phase noise measurements and the simulations of EVM performance at different phase noise levels, of the two evaluated schemes only the MZM based scheme can match the phase noise performance required for 


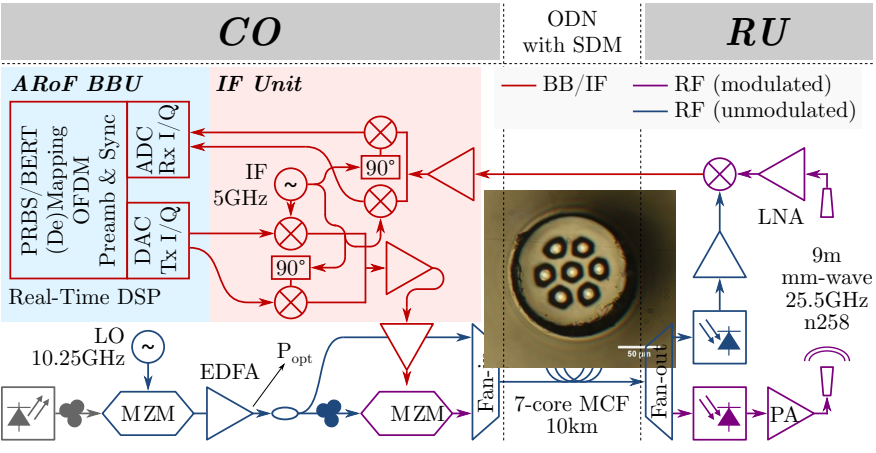

Fig. 8. Experimental setup of ARoF transmission with real-time signal processing, $26.5 \mathrm{GHz} \mathrm{mm}$-wave wireless over $9 \mathrm{~m}$ and the remote-fed LO.

real-time processing in the AROF BBU and the latter is thus employed in the following experimental demonstration. While the current OPLL implementation can not reach the required phase noise levels, the authors predict that with an optimized selection of lasers and design of the feedback loop, phase noise levels sufficiently low to allow operation with the AROF BBU can be achieved. With the given lasers, the $1 / f^{2}$ slope of the free running beating tone should be considered as a fundamental limit and it is expected that an extension of OPLL bandwidth by one decade would lower the phase noise plateau by $20 \mathrm{dBc} / \mathrm{Hz}$, as shown in the phase noise profiles in Fig. 7. While OPLL bandwidth is limited by the change of tuning mechanism of the DFB laser between thermal (low frequencies, opposite phase to frequency modulation) and carrier (high frequencies, in phase) which occurs in the $\mathrm{MHz}$ range, the inclusion of a phase advance in the loop filter to anticipate for this change can allow extension of the loop bandwidth by several MHz.

\section{Millimeter-Wave 5G Performance with Analog RADIO-OVER-FIBER FRONTHAUL}

In order to validate the basic AROF setup previously shown at the top of Fig. 2 and to evaluate the achieved performance under the given phase noise levels, the experimental setup in Fig. 8 is employed [16]. The setup further serves to validate the time DSP of the AROF BBU and to showcase the bandwidth achievable in ARoF systems by transmitting an extended $5 \mathrm{G}$ NR OFDM signal of $800 \mathrm{MHz}$ bandwidth. The implemented AROF link features the blueSPACE AROF BBU, IF unit and an ARoF transmitter based on bulk optical components at the CO, as well as commercially available RF amplifiers and antennas at the RU for mm-wave wireless transmission and electrical downconversion based on the remote-fed LO. It should be noted that no UE is included in the experiment and that the transmitted signal of the RU downlink is also its received signal of the RU uplink.

The AROF BBU generates extended 5G NR signals with a total of 4096 subcarriers, spaced at $240 \mathrm{kHz}$, of which 3136 are active, resulting in an effective signal bandwidth of $760.32 \mathrm{MHz}$. The chosen modulation for the subcarriers is quadrature phase-shift keying (QPSK), resulting in a data rate of $1.4 \mathrm{Gbit} / \mathrm{s}$. The BBU further includes DAC and ADC, generating and receiving separate in-phase and quadrature (IQ) base- band signals respectively. Frequency up- and downconversion between baseband and IF is performed by the IF unit, employing a single tuneable $\mathrm{LO}$ at $2.25 \mathrm{GHz} \leq f_{\mathrm{IF}, \mathrm{LO}} \leq 5.5 \mathrm{GHz}$ and a pair of IQ modulator and demodulator; throughout this experiment, $f_{\mathrm{IF}, \mathrm{LO}}$ remains fixed at $5 \mathrm{GHz}$.

Optical two-tone generation is performed by a MZM biased at the null point and driven with an $\mathrm{LO}$ at $f_{\mathrm{RF} / 2, \mathrm{LO}}=$ $10.25 \mathrm{GHz}$, resulting in a spacing of $20.5 \mathrm{GHz}$ between the two optical tones [11]. The two-tone signal is amplified and split in two equal branches, one is directly transmitted via the MCF to the RU, where, after amplification by $30 \mathrm{~dB}$, it serves as LO at $f_{\mathrm{RF}, \mathrm{LO}}=20.5 \mathrm{GHz}$ for subsequent electrical downconversion of the received mm-wave signal. The other is modulated with the IF signal in a second MZM, before being transmitted to the RU via a separate core of the $10 \mathrm{~km} \mathrm{7-core}$ MCF. Here the use of MCF allows the seamless transport of both signals, despite being at the same wavelength.

The mm-wave RF signal is generated by optical heterodyning of the modulated two-tone signal on the PD at the $\mathrm{RU}$, resulting in a signal that includes modulated components at $f_{\mathrm{RF}, \mathrm{low}}=f_{\mathrm{RF}, \mathrm{LO}}-f_{\mathrm{IF}}=15.5 \mathrm{GHz}$ and at $f_{\mathrm{RF}}=$ $f_{\mathrm{RF}, \mathrm{LO}}+f_{\mathrm{IF}}=25.5 \mathrm{GHz}$, as well as other lower frequency components. The latter as well as the unwanted signal at $f_{\mathrm{RF} \text {,low }}$ are removed by a high-pass filter (HPF), before the signal is amplified by $30 \mathrm{~dB}$ using a medium power amplifier (MPA) and wirelessly transmitted at $25.5 \mathrm{GHz}$ - i.e., in the 3 GPP n258 band [28] - over $9 \mathrm{~m}$. Wireless transmission is performed using a pair of horn antennas with a gain of $18.5 \mathrm{dBi}$ each. The received mm-wave signal is amplified by $40 \mathrm{~dB}$ with a LNA before being downconverted to IF and fed back to the IF unit and AROF BBU.

The evaluation of the ARoF fronthaul link is based on the spectra of the transmitted RF signal and the transmitted and received IF signals, as well as on the real-time bit error rate (BER) and corresponding signal constellations. Figure 9(a) shows the transmitted RF spectrum, clearly showing the targeted $25.5 \mathrm{GHz}$ signal, as well as a tone at $f_{\mathrm{RF}, \mathrm{LO}}$ resulting from the beating of the two optical tones. The latter is suppressed by around $20 \mathrm{~dB}$ compared to the target carrier by the HPF and as it is outside the antenna band is not transmitted. Figure 9(b) shows a comparison of the transmitted and received IF signals centered at $5 \mathrm{GHz}$. While both signals are at roughly equal power, the received IF signal has a severely degraded SNR of around $12 \mathrm{~dB}$, compared to $>30 \mathrm{~dB}$ for the transmitted IF signal. The received IF spectrum further has a slightly lower carrier-to-signal ratio as well as a tilt towards the higher frequencies, suggesting a non-flat system response across signal bandwidth at IF or RF.

System BER performance was evaluated for different optical powers after the two-tone generation, i.e., at the output of the first EDFA in the AROF transmitter. In the given setup, every $1 \mathrm{~dB}$ of optical power reduction translates directly into $2 \mathrm{~dB}$ power reduction for both mm-wave RF and LO signals at the $\mathrm{RU}$, thus an estimated $5 \mathrm{~dB}$ in received IF signal degradation - where the additional $1 \mathrm{~dB}$ is due to lower mixer efficiency at lower LO drive levels. The evaluated range of optical powers thus translates into an RF power variation of $4 \mathrm{~dB}$ and a variation of the received IF power by $10 \mathrm{~dB}$. BER is 


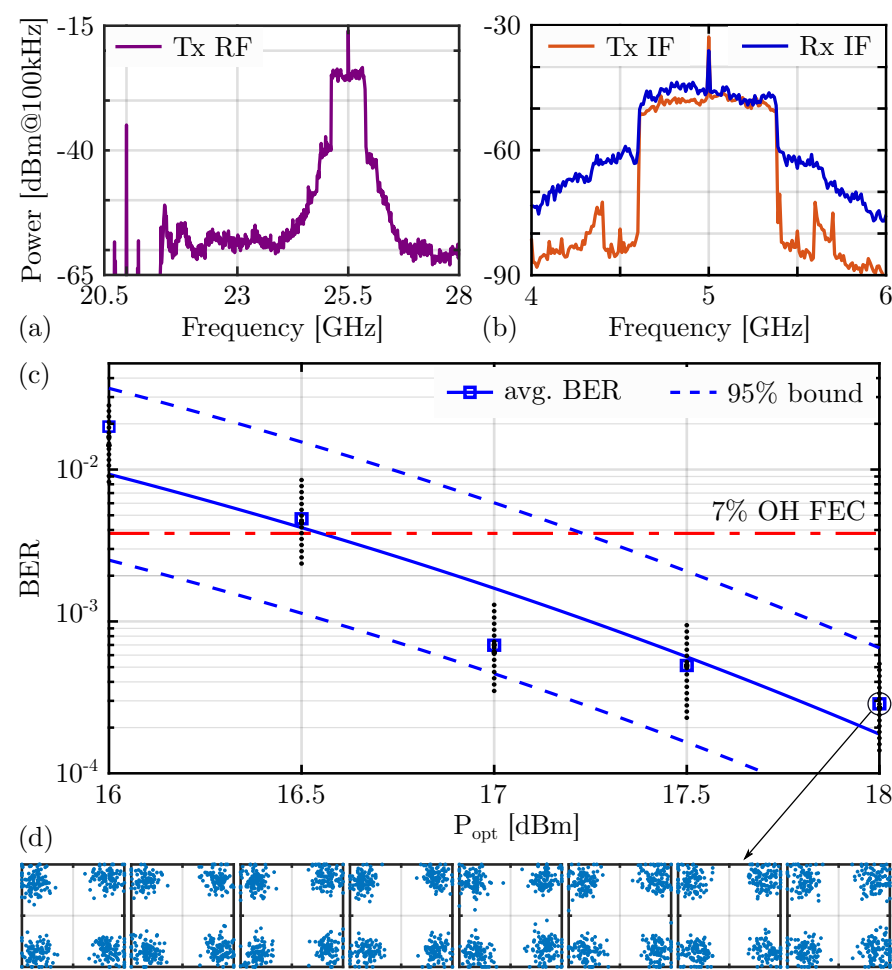

Fig. 9. Measurement results for the real-time ARoF and mm-wave transmission: a) spectrum of the transmitted RF signal, b) spectra of the transmitted and received IF signals, c) BER over optical power of the two-tone signal, d) received constellations for eight equal size subcarrier groups.

measured repeatedly over short time intervals of a few seconds at each power level, to not only capture average BER, but also its variation over time. The resulting BER measurements as well as their averages are shown in Fig. 9(c), alongside the BER limit for a standard forward error correction (FEC) with $7 \%$ overhead. The figure further shows the $95 \%$ confidence bounds for the BER for any such short time interval, derived from the measured BER statistics.

Finally, Fig. 9(d) shows the received QPSK constellations, splitting the $800 \mathrm{MHz}$ wide signals into eight subcarrier groups of equal width of roughly $100 \mathrm{MHz}$. As can be seen, the constellations are similar for all groups, with mainly the first and the two last groups somewhat degraded. This is likely due to reduced low-frequency performance of the modulator in the IF unit as well as bandwidth limitations of the latter and the slight power reduction at higher frequencies previously observed on the received IF signal.

While the observed BER performance and constellations show the presented AROF fronthaul and mm-wave transmission link to easily reach the BER limit for a simple hard-decision FEC with some power margin, it should not be taken to represent the ultimate performance that can be achieved by such a system. Performance of the presented system was limited by low power on the PD at the RU and the LNA approaching saturation due to the fixed-gain nature of the employed mm-wave amplifiers and the available transmission distance in the laboratory. In a system implementation with improved optical power budget and adjustable gain mm-wave amplifiers and under the inclusion of optical beamforming the use of higher-order modulation is expected to be achievable, significantly improving spectral efficiency.

\section{CONCLUSIONS}

This article discussed the use of ARoF for the fronthaul of high-bandwidth mm-wave 5G NR signals, briefly introducing how ARoF fronthaul systems can address the fronthaul capacity crunch faced by traditional CPRI fronthaul, while avoiding a partial return to a distributed RAN with some signal processing at the antenna sites. The blueSPACE architecture for an ARoF fronthaul system with optical beamforming was introduced and two corresponding multi-beam ARoF transmitters designs were discussed. To lay the foundation for a full implementation, the phase noise performance of two ARoF schemes was measured and the tolerance of the real-time signals processing in the AROF BBU was investigated. An AROF fronthaul link was implemented, experimentally demonstrating real-time transmission of mm-wave extended $5 \mathrm{G}$ NR signals with MCFbased fronthaul and wireless transmission at $25.5 \mathrm{GHz}$.

The proposed blueSPACE ARoF fronthaul architecture features an LO fed from the $\mathrm{CO}$ to the $\mathrm{RU}$ for maximized centralization and includes OBFNs to allow true multi-beam transmission from a single antenna array. ARoF two-tone generation with a SC-MZM or an OPLL are described in detail, including designs for integrated multi-channel ARoF transmitters as building blocks towards the introduction of optical beamforming. The phase noise performance of the two ARoF transmitter schemes was experimentally evaluated, showing the SC-MZM approach to match the phase noise of the underlying RF source, while the OPLL successfully suppresses part of the combined phase noise of the two lasers, but with overall higher phase noise. The maximum acceptable phase noise level for operation with the real-time ARoF BBU implemented in blueSPACE was investigated through simulations, suggesting that operation with an improved implementation of the OPLL is feasible.

The experimental demonstration of AROF fronthaul over MCF with a remote-fed LO for downconversion at the RU validates the proposed basic ARoF architecture, as well as the feasibility of AROF fronthaul with optical heterodyning for high-bandwidth 5G NR signals. Successful real-time transmission of extended $5 \mathrm{G}$ NR signals of $800 \mathrm{MHz}$ bandwidth with an OFDM configuration following the 3GPP numerology was shown over $10 \mathrm{~km} \mathrm{7-core} \mathrm{MCF} \mathrm{and} 9 \mathrm{~m} \mathrm{~mm}$-wave wireless transmission at $25.5 \mathrm{GHz}$. The demonstrated link achieved BERs below the limit for a commercial FEC with $7 \%$ overhead at a data rate of $1.4 \mathrm{Gbit} / \mathrm{s}$.

Together these serve to set the requirements for a full implementation of the blueSPACE architecture with integrated AROF transmitters and OBFNs and to demonstrate the feasibility of high-bandwidth ARoF fronthaul with MCF transport. The combination of ARoF fronthaul with optical beamforming, supported by integrated AROF transmitters, SDM optical transport and the AROF BBU and IF unit, shows the way to a highly flexible and widely scaleable fronthaul solution for mm-wave 5G NR signals supporting bandwidths beyond those proposed in current standardization efforts. 


\section{REFERENCES}

[1] J. G. Andrews et al., "What will 5G be?" IEEE J. Sel. Areas Commun., vol. 32, no. 6, pp. 1065-1082, Jun. 2014.

[2] ITU-R M.2083, "IMT vision - framework and overall objectives of the future development of IMT for 2020 and beyond," Sep. 2015.

[3] T. Pfeiffer, "Next generation mobile fronthaul and midhaul architectures," J. Opt. Commun. Netw., vol. 7, no. 11, pp. B38-B45, Sep. 2015.

[4] S. Rommel et al., "High-capacity 5G fronthaul networks based on optical space division multiplexing," IEEE Trans. Broadcast., vol. 65, no. 2, pp. 434-443, Jun. 2019.

[5] P. Rost et al., "Mobile network architecture evolution toward 5G," IEEE Commun. Mag., vol. 54, no. 5, pp. 84-91, May 2016.

[6] S. Rommel, T. R. Raddo, and I. Tafur Monroy, "The fronthaul infrastructure of 5G mobile networks," in IEEE Intl. Workshop Computer-Aided Model. Anal. and Des. Commun. Links Netw. (CAMAD), Sep. 2018, paper S4.4.

[7] T. S. Rappaport et al., "Millimeter wave mobile communications for 5G cellular: It will work!" IEEE Access, vol. 1, pp. 335-349, 2013.

[8] M. Xiao et al., "Millimeter wave communications for future mobile networks," IEEE J. Sel. Areas Commun., vol. 35, no. 9, pp. 1909-1935, Sep. 2017.

[9] J. Liu, M. Sheng, L. Liu, and J. Li, "Network densification in 5G: From the short-range communications perspective," IEEE Commun. Mag., vol. 55, no. 12, pp. 96-102, Dec. 2017.

[10] W. Feng et al., "When mmWave communications meet network densification: A scalable interference coordination perspective," IEEE J. Sel. Areas Commun., vol. 35, no. 7, pp. 1459-1471, Jul. 2017.

[11] S. Rommel et al., "W-band photonic-wireless link with a Schottky diode envelope detector and bend insensitive fiber," Opt. Express, vol. 24, no. 11, pp. 11312-11322, May 2016.

[12] A. Kanno et al., "Seamless fiber-wireless bridge in the millimeter- and terahertz-wave bands," J. Lightw. Technol., vol. 34, no. 20, pp. 4794 4801, Oct. 2016.

[13] J. Yao, "Microwave photonics," J. Lightw. Technol., vol. 27, no. 3, pp. 314-335, Feb. 2009.

[14] M. Hinrichs, L. F. Del Rosal, C. Kottke, and V. Jungnickel, "Analog vs. next-generation digital fronthaul: How to minimize optical bandwidth utilization," in Intl. Conf. Opt. Netw. Des. Model. (ONDM), Jun. 2017.

[15] C. Mitsolidou et al., "A 5G C-RAN optical fronthaul architecture for hotspot areas using OFDM-based analog IFoF waveforms," Appl. Sci., vol. 9, no. 19, p. 4059, Sep. 2019.

[16] J. Brenes et al., "Network slicing architecture for SDM and analogradio-over-fiber-based 5G fronthaul networks," J. Opt. Commun. Netw., vol. 12, no. 4, pp. B33-B43, Apr. 2020.

[17] J. Pérez Santacruz et al., "Candidate waveforms for $\mathrm{ARoF}$ in beyond 5G," Appl. Sci., vol. 10, no. 11, p. 3891, Jun. 2020.

[18] A. Kanno et al., "Evaluation of frequency fluctuation in fiber-wireless link with direct IQ down-converter," in Europ. Conf. Opt. Commun. (ECOC), Sep. 2014, paper We.3.6.3.

[19] S. Rommel et al., "Outdoor W-band hybrid photonic wireless link based on an optical SFP+ module," IEEE Photon. Technol. Lett., vol. 28 , no. 21, pp. 2303-2306, Nov. 2016.

[20] K. Van Gasse et al., "Analog radio-over-fiber transceivers based on IIIV-on-silicon photonics," IEEE Photon. Technol. Lett., vol. 30, no. 21 , pp. 1818-1821, Nov. 2018.

[21] C. Roeloffzen et al., "Enhanced coverage though optical beamforming in fiber wireless networks," in Intl. Conf. Transpar. Opt. Netw. (ICTON), Jul. 2017, paper TH.A2.1.

[22] D. Konstantinou et al., "5G RAN architecture based on analog radioover-fiber fronthaul over UDWDM-PON and phased array fed reflector antennas," Opt. Commun., vol. 454, p. 124464, Jan. 2020.

[23] W. Klaus et al., "Advanced space division multiplexing technologies for optical networks," J. Opt. Commun. Netw., vol. 9, no. 4, pp. C1-C11, Apr. 2017.

[24] A. Macho, M. Morant, and R. Llorente, "Next-generation optical fronthaul systems using multicore fiber media," J. Lightw. Technol., vol. 34, no. 20, pp. 4819-4827, Oct. 2016.

[25] J. M. Galve, I. Gasulla, S. Sales, and J. Capmany, "Reconfigurable radio access networks using multicore fibers," IEEE J. Quantum Electron., vol. 52, no. 1, p. 0600507, Jan. 2016.

[26] blueSPACE Consortium, "Space division multiplexing 5G fronthaul with analog and digital radio-over-fiber and optical beamforming - the blueSPACE concept," Aug. 2018. [Online]. Available: https: //doi.org/10.5281/zenodo.1403140
[27] S. Rommel et al., "Real-time demonstration of ARoF fronthaul for highbandwidth mm-wave 5G NR signal transmission over multi-core fiber," in Europ. Conf. Netw. Commun. (EuCNC), Jun. 2020, pp. 225-228.

[28] 3GPP TS 38.104, "NR: Base station (BS) radio transmission and reception," Mar. 2018, 3GPP Rel. 15.

[29] A. Checko et al., "Cloud RAN for mobile networks-a technology overview," IEEE Commun. Surveys Tuts., vol. 17, no. 1, pp. 405-426, 2015.

[30] L. M. Larsen, A. Checko, and H. L. Christiansen, "A survey of the functional splits proposed for 5G mobile crosshaul networks," IEEE Commun. Surveys Tuts., vol. 21, no. 1, pp. 146-172, Jan. 2019.

[31] J. Beas et al., "Millimeter-wave frequency radio over fiber systems: A survey," IEEE Commun. Surveys Tuts., vol. 15, no. 4, pp. 1593-1619, 2013.

[32] J. J. O'Reilly, P. M. Lane, R. Heidemann, and R. Hofstetter, "Optical generation of very narrow linewidth millimetre wave signals," Electron. Lett., vol. 28, no. 25, pp. 2309-2311, 1992.

[33] T. J. Kippenberg, R. Holzwarth, and S. A. Diddams, "Microresonatorbased optical frequency combs," Science, vol. 332, no. 6029, pp. 555559, Apr. 2011.

[34] P. J. Delfyett et al., "Optical frequency combs from semiconductor lasers and applications in ultrawideband signal processing and communications," J. Lightw. Technol., vol. 24, no. 7, pp. 2701-2719, Jul. 2006.

[35] L. Langley et al., "Packaged semiconductor laser optical phase-locked loop (OPLL) for photonic generation, processing and transmission of microwave signals," IEEE Trans. Microw. Theory Tech., vol. 47, no. 7, pp. 1257-1264, Jul. 1999.

[36] S. Ristic et al., "An optical phase-locked loop photonic integrated circuit," J. Lightw. Technol., vol. 28, no. 4, pp. 526-538, Feb. 2009.

[37] K. Balakier et al., "Integrated semiconductor laser optical phase lock loops," IEEE J. Sel. Topics Quantum Electron., vol. 24, no. 1, p. 1500112, Jan. 2017

[38] A. Yariv, "Dynamic analysis of the semiconductor laser as a currentcontrolled oscillator in the optical phased-lock loop: applications," Optics Lett., vol. 30, no. 17, pp. 2191-2193, Sep. 2005.

[39] F. M. Gardner, Phaselock techniques. John Wiley \& Sons, 2005.

[40] H. Schippers et al., "Broadband conformal phased array with optical beam forming for airborne satellite communication," in IEEE Aerospace Conf., 2008.

[41] C. G. Roeloffzen et al., "Integrated optical beamformers," in Opt. Fiber Commun. Conf. (OFC), 2015, paper Tu3F-4.

[42] A. Meijerink et al., "Novel ring resonator-based integrated photonic beamformer for broadband phased array receive antennas-Part I: Design and performance analysis," J. Lightw. Technol., vol. 28, no. 1, pp. 3-18, Jan. 2010.

[43] L. Zhuang et al., "Novel ring resonator-based integrated photonic beamformer for broadband phased array receive antennas-Part II: Experimental prototype," J. Lightw. Technol., vol. 28, no. 1, pp. 19-31, Jan. 2010.

[44] M. Smit et al., "An introduction to InP-based generic integration technology," Semicond. Sci. Technol, vol. 29, no. 8, p. 083001, Aug. 2014.

[45] C. G. H. Roeloffzen et al., "Low-loss Si3N4 TriPleX optical waveguides: Technology and applications overview," IEEE J. Sel. Topics Quantum Electron., vol. 24, no. 4, p. 4400321, Jul. 2018.

[46] J. M. Ramirez et al., "III-V-on-silicon integration: From hybrid devices to heterogeneous photonic integrated circuits," IEEE J. Sel. Topics Quantum Electron., vol. 26, no. 2, p. 6100213, 2019.

[47] J. P. Epping et al., "High power, tunable, narrow linewidth dual gain hybrid laser," in Laser Congress, 2019, paper ATu1A.4.

[48] S. Mosca, F. Bilotti, A. Toscano, and L. Vegni, "A novel design method for Blass matrix beam-forming networks," IEEE Trans. Antennas Propag., vol. 50, no. 2, pp. 225-232, Feb. 2002.

[49] F. Azendorf et al., "Characterization of multi-core fiber group delay with correlation otdr and modulation phase shift methods," in Opt. Fiber Commun. Conf. (OFC), 2020, paper M2C.5.

[50] G. Qi et al., "Phase-noise analysis of optically generated millimeterwave signals with external optical modulation techniques," J. Lightw. Technol., vol. 24, no. 12, pp. 4861-4875, Dec. 2006.

[51] I. Ngebani et al., "Analysis and compensation of phase noise in vector OFDM systems," IEEE Trans. Signal Process., vol. 62, no. 23, pp. 6143 6157, Dec. 2014.

[52] A. Delmade et al., "OFDM baud rate limitations in an optical heterodyne analog fronthaul link using unlocked fibre lasers," in IEEE Intl. Top. Meet. Microw. Photon. (MWP), Oct. 2019. 